\title{
A Nash type solution for hemivariational inequality systems Systems
}

\author{
Dušan Repovš and Csaba Varga \\ ${ }^{c}$ Faculty of Mathematics and Physics, and Faculty of Education, University of Ljubljana, \\ P. O. B. 2964, Ljubljana, Slovenia 1001 \\ Babeş-Bolyai University, Faculty of Mathematics and Computer Sciences, RO-400084 Cluj-Napoca, \\ Romania \\ Email address: dusan.repovs@guest.arnes.si and csvarga@cs.ubbcluj.ro
}

\begin{abstract}
In this paper we prove an existence result for a general class of hemivariational inequalities systems using the Ky Fan version of the KKM theorem (1984) or the Tarafdar fixed point theorem (1987). As application we give an infinite dimensional version for existence result of Nash generalized derivative points introduced recently by Kristály (2010) and also we give an application to a general hemivariational inequalities system.
\end{abstract}

Key words: Fixed point theory, nonsmooth functions, hemivariational inequality systems.

2000 Mathematics Subject Classification: 35B34; 47J20; 58E05.

\section{Introduction}

In the last years many papers have been dedicated to the of study the existence and multiplicity of solutions for hemivariational inequality systems or differential inclusion systems defined on bounded or unbounded domain, see [2], [3, [11, [12], 13], [16]. In these papers the authors use critical points theory for locally Lipschitz functions, combined with the Principle of Symmetric Criticality and different topological methods. For a comprehensive treatment of hemivariational inequality and hemivariational inequalities systems on bounded domains using critical point theory for nonsmooth functionals we refer to the monographs of D. Motreanu and V. Rădulescu [17] and D. Motreanu and P. D. Panagiotopoulos [18. For very recent results concerning variational inequalities and elliptic systems using critical points theory and different variational methods see also the book of Kristály, Rădulescu and Varga [15].

The aim of this paper is to prove that the existence of at least one solution for a general class of hemivariational inequalities systems on a closed and convex set (either bounded or unbounded) without using critical point theory. We apply a version of the well-known theorem of KnasterKuratowski-Mazurkiweicz due to Ky Fan [7] or the Tarafdar fixed point theorem [23]. We start the paper by giving in Section 2 the assumptions and formulating the hemivariational inequalities system problem which we study. The main results concerning the existence of at least one solution for the hemivariational inequalities systems which we study are given in Section 3. Section 4 contains applications to Nash and Nash general derivative points and existence results for some abstract class of hemivariational inequalities systems. 


\section{Assumptions and Formulation of the Problem}

Let $X_{1}, X_{2}, \ldots, X_{n}$ be reflexive Banach spaces and $Y_{1}, Y_{2}, \ldots, Y_{n}, Z_{1}, \ldots, Z_{n}$ Banach spaces such that there exist linear operators $T_{i}: X_{i} \rightarrow Y_{i}, T_{i}: X_{i} \rightarrow Z_{i}$ for $i \in\{1, \ldots, n\}$. We suppose that the following condition hold:

(TS) $T_{i}: X_{i} \rightarrow Y_{i}$ and $S_{i}: X_{i} \rightarrow Z_{i}$ are compact for $i=\overline{1, n}$.

We denote by $X_{i}^{*}$ the topological dual of $X_{i}$ and $\langle\cdot, \cdot\rangle_{i}$ denotes the duality pairing between $X_{i}^{*}$, whereas $X_{i}$ for $i=\overline{1, n}$. Also, let $K_{i} \subset X_{i}$ be closed, convex sets for $i=\overline{1, n}$ and we consider $A_{i}: Y_{1} \times \cdots \times Y_{i} \times \cdots \times Y_{n} \rightarrow \mathbb{R}$ the continuous functions which are locally Lipschitz in the $i$ th variable and we denote by $A_{i}^{\circ}\left(u_{1}, \ldots, u_{i}, \ldots, u_{n} ; v_{i}\right)$ the partial Clarke derivative in the directional derivative in the $i$ th variable, i.e. the Clarke derivative of the locally Lipschitz function $A_{i}\left(u_{1}, \ldots, u_{i}, \ldots, u_{n}\right)$ at the point $u_{i} \in Y_{i}$ in the direction $v_{i} \in Y_{i}$, that is

$$
A_{i}^{\circ}\left(u_{1}, \ldots, u_{i}, \ldots u_{n} ; v_{i}\right)=\limsup _{\substack{w \rightarrow u_{i} \\ \tau \searrow 0}} \frac{A_{i}\left(u_{1}, \ldots, w+\tau v_{i}, \ldots u_{n}\right)-A_{i}\left(u_{1}, \ldots, w, \ldots u_{n}\right)}{\tau}
$$

We suppose that for every $i=\overline{1, n}$ the following condition holds:

(A) the functions $A_{i}^{\circ}: Y_{1} \times \cdots \times Y_{n} \times Y_{i} \rightarrow \mathbb{R}$ are upper semi-continuous.

We also consider the following nonlinear operators $F_{i}: K_{1} \times \cdots \times K_{i} \times \cdots K_{n} \rightarrow X_{i}^{\star}, i=\overline{1, n}$. We suppose that the operators $F_{i}$ satisfy the following condition:

(F) the functions $\left(u_{1}, \ldots, u_{n}\right) \mapsto\left\langle F_{i}\left(u_{1}, \ldots, u_{n}\right), v_{i}\right\rangle_{i}$ are weakly upper semi-continuous for every $v_{i} \in X_{i}$ and $i=\overline{1, n}$.

Definition 2.1 [see [5] Let $Z$ be a Banach space and $j: Z \rightarrow \mathbb{R}$ a locally Lipschitz function. We say that $j$ is regular at $u \in Z$ if for all $v \in Z$ the one-sided directional derivative $j^{\prime}(u ; v)$ exists and $j^{\prime}(u ; v)=j^{\circ}(u ; v)$. If $j$ is regular at every point $u \in Z$ we say that $j$ is regular.

We have the following elementary result.

Proposition 2.1 Let $J: Z_{1} \times \cdots \times Z_{n} \rightarrow \mathbb{R}$ be a regular, locally Lipschitz function. Then the following assertions hold:

a) $\partial J\left(u_{1}, \ldots, u_{n}\right) \subseteq \partial_{1} J\left(u_{1}, \ldots, u_{n}\right) \times \cdots \times \partial_{n} J\left(u_{1}, \ldots, u_{n}\right)$ (see [5], Proposition 2.3.15), where $\partial_{i} J, i=\overline{1, n}$ denotes the Clarke subdifferential in the ith variable;

b) $J^{\circ}\left(u_{1}, \ldots, u_{n} ; v_{1}, \ldots, v_{n}\right) \leq \sum_{i=1}^{n} J_{i}^{\circ}\left(u_{1}, \ldots, u_{n} ; v_{i}\right)$, where $J_{i}^{\circ}$ denotes the Clarke derivative in the ith variable; and

c) $J^{\circ}\left(u_{1}, \ldots, u_{n} ; 0, \ldots, v_{i}, \ldots, 0\right) \leq J_{i}^{\circ}\left(u_{1}, \ldots, u_{n} ; v_{i}\right)$.

We introduce the following notations:

- $K=K_{1} \times \cdots \times K_{n}$,

- $u=\left(u_{1}, \ldots, u_{n}\right)$

- $T u=\left(T_{1} u_{1}, \ldots, T_{n} u_{n}\right)$

- $S u=\left(S_{1} u_{1}, \ldots, S_{n} u_{n}\right)$

- $A(T u, T v-T u)=\sum_{i=1}^{n} A_{i}^{\circ}\left(T u, T_{i} v_{i}-T_{i} u_{i}\right)$

- $F(u, v-u)=\sum_{i=1}^{n}\left\langle F_{i} u, v_{i}-u_{i}\right\rangle_{i}$ 
In this paper we study the following problem:

Find $u=\left(u_{1}, \ldots, u_{n}\right) \in K_{1} \times \ldots \times K_{n}$ such that for all $v=\left(v_{1}, \ldots, v_{n}\right) \in K_{1} \times \ldots \times K_{n}$ and $i \in\{1, \ldots, n\}$ we have:

(QHS) $\quad A_{i}^{\circ}\left(T u ; T v_{i}-T u_{i}\right)+\left\langle F_{i}(u), v_{i}-u_{i}\right\rangle_{i}+J_{i}^{\circ}\left(S u ; S_{i} v_{i}-S_{i} u_{i}\right) \geq 0$.

In this case we say that $u=\left(u_{1}, \ldots, u_{n}\right)$ is a Nash equilibrum point for the system (QHS).

To prove our main result we use the FKKM theorem due to Ky Fan [7] and the Tarafdar fixed point theorem [23].

Definition 2.2 Suppose that $X$ is a vector space and $E \subset X$. A set-valued mapping $G: E \rightarrow 2^{X}$ is called a KKM mapping, if for any $x_{1}, \ldots, x_{n} \in E$ the following holds

$$
\operatorname{conv}\left\{x_{1}, \ldots, x_{n}\right\} \subset \bigcup_{i=1}^{n} G\left(x_{i}\right) .
$$

The following version of the KKM theorem is due to Ky Fan [7.

Theorem 2.1 Suppose that $X$ is a locally convex Hausdorff space, $E \subset X$ and that $G: E \rightarrow 2^{X}$ is a closed-valued KKM map. If there exists $x_{0} \in E$ such that $G\left(x_{0}\right)$ is compact, then $\bigcap_{x \in E} G(x) \neq \emptyset$.

Theorem 2.2 Let $K$ be a nonempty, convex subset of a Hausdorff topological vector space $X$. Let $G: K \hookrightarrow 2^{K}$ be a setvalued map such that

i) for each $u \in K, G(u)$ is a nonemty convex subset of $K$;

ii) for each $v \in K, G^{-1}(v)=\{u \in K \quad: v \in G(u)\}$ contains an open set $O_{v}$ which may be empty;

iii) $\cup_{v \in K} O_{v}=K$; and

iv) there exists a nonemty set $K_{0}$ contained in a compact convex subset $K_{1}$ of $K$ such that $D=\cap_{v \in K_{0}} O_{v}^{c}$ is either empty or compact (where $O_{v}^{c}$ is the complement of $O_{v}$ in $K$ ).

Then there exists a point $u_{0} \in K$ such that $u_{0} \in G\left(u_{0}\right)$.

E. Tarafdar in [23] proved the equivalence of Theorems 2.1 and 2.2

\section{Main results}

Theorem 3.1 Let $K_{i} \subset X_{i}, i=\overline{1, n}$ be nonempty, bounded, closed and convex sets. Let $A_{i}$ : $Y_{1} \times \cdots \times Y_{i} \times \cdots \times Y_{n} \rightarrow \mathbb{R}$ be a locally Lipschitz function in the $i$ th variable for all $i \in\{1, \ldots, n\}$ satisfying condition (A). We suppose that the operators $T_{i}: X_{i} \rightarrow Y_{i}, S_{i}: X_{i} \rightarrow Z_{i}$ and $F_{i}$ : $K_{1} \times \ldots \times K_{n} \rightarrow X_{i}^{\star}(i=\overline{1, n})$ satisfy the condition (TS) respectively $(\mathbf{F})$. Final we consider the regular locally Lipschitz function $J: Z_{1} \times \cdots \times Z_{n} \rightarrow \mathbb{R}$. Under these conditions the problem (QHS) admits at least one solution.

Before proving Theorem 3.1, we make two remarks.

Remark 3.1 We observe that for every $v \in K$ the function

$$
u \mapsto A(T u, T v-T u)+F(u, v-u)+J^{\circ}(S u ; S v-S u)
$$

is weakly upper semi-continuous. Indeed, from the condition (A) and from the fact that the operators $T_{i}$ are compact follows that $A(T u, T v-T u)$ is weakly upper semi-continuous. From $(\mathbf{F})$ it follows that $F(u, v-u)$ is weakly upper semi-continuous. The third term, i.e. $J^{\circ}(S u ; S v-S u)$ is weakly upper semi-continuous, because $J^{\circ}(\cdot ; \cdot)$ is upper semi-continuous and the operators $S_{i}: X_{i} \rightarrow Z_{i}$ are compact. 
Remark 3.2 If there exists $u \in K$, such that for every $v \in K$ we have:

$$
A(T u, T v-T u)+F(u, v-u)+J^{\circ}(S u ; S v-S u) \geq 0,
$$

then $u \in K$ is a solution of the problem (QHS). Indeed, if we fix an $i=\{1, \ldots, n\}$ and put $v_{j}:=u_{j}, j \neq i$ in the above inequality and using iii) Proposition [2.1 we get that

$(Q H S), \quad A_{i}^{\circ}\left(T u ; T v_{i}-T u_{i}\right)+\left\langle F_{i}\left(u_{i}\right), v_{i}-u_{i}\right\rangle_{i}+J_{i}^{\circ}\left(S u ; S_{i} v_{i}-S_{i} v_{i}\right) \geq 0$.

for all $i \in\{1, \ldots, n\}$.

In the sequel we give two proofs, using Theorems 2.1 and 2.2 .

First Proof: Let $G: K \hookrightarrow 2^{K}$ be the set-valued map defined by

$$
G(v)=\left\{u \in K: A(T u, T v-T u)+F(u, v-u)+J^{\circ}(S u ; S v-S u) \geq 0\right\} .
$$

For every $v \in K$, we have $G(v) \neq \emptyset$ because $v \in G(v)$ and taking into account that the function

$$
u \mapsto A(T u, T v-T u)+F(u, v-u)+J^{\circ}(S u ; S v-S u)
$$

is weakly upper semi-continuous, it follows that the set $G(v)$ is weakly closed. Now we prove that $G$ is a KKM mapping. We argue by contradiction, let $v_{1}, \ldots, v_{k} \in K$ and $w \in \operatorname{conv}\left\{v_{1}, \ldots, v_{k}\right\}$ such that $w \notin \cup_{i=1}^{k} G\left(v_{i}\right)$. From this it follows that

$$
A\left(T w, T v_{i}-T w\right)+F\left(w, v_{i}-w\right)+J^{\circ}\left(S w ; S v_{i}-S w\right)<0,
$$

for all $i=\{1, \ldots, k\}$. Because of $w \in \operatorname{conv}\left\{v_{1}, \ldots, v_{k}\right\}$ the existence of $\lambda_{1}, \ldots, \lambda_{k} \in[0,1]$ with $\sum_{i=1}^{k} \lambda_{i}=1$ such that $w=\sum_{i=1}^{k} \lambda_{i} v_{i}$ follows . If we multiply the inequalities (3.2) with $\lambda_{i}$ and adding for $i=\{1, \ldots, k\}$ we obtain

$$
A(T w, T w-T w)+F(w, w-w)+J^{\circ}(S w ; S w-S w)<0
$$

because the functions $A(\cdot, \cdot), F(\cdot, \cdot)$ and $J^{\circ}(\cdot, \cdot)$ are positive homogeneous and convex in the second variable. From inequality (3.3) it follows that $0=A(T w, T w-T w)+F(w, w-w)+J^{\circ}(S w ; S w-$ $S w)<0$, which is a contradiction. Because the set $K$ is bounded, convex and closed, it follows that it is weakly closed and by the Eberlein-Smulian theorem we have is weakly compact. Because $G(v) \subset K$ is weakly closed, we have that $G(v)$ is weakly compact and from Theorem 2.1 it follows that $\cap_{v \in K} G(v) \neq \emptyset$, therefore from Remark 3.2 it follows that the problem (QHS) has a solution.

Second Proof. Using Remark 3.2 we prove the existence of an element $u \in K$ such that for every $v \in K$ we have

$$
A(T u, T v-T u)+F(u, v-u)+J^{\circ}(S u ; S v-S u) \geq 0 .
$$

In this case $u \in K$ will be the solution of systems (QHS).

We argue by contradiction. Let us assume that for each $u \in K$, there exists $v \in K$ such that

$$
A(T u, T v-T u)+F(u, v-u)+J^{\circ}(S u ; S v-S u)<0 .
$$

Now, we define the set-valued mapping $G: K \hookrightarrow 2^{K}$ by

$$
G(u)=\left\{v \in K: A(T u, T v-T u)+F(u, v-u)+J^{\circ}(S u ; S v-S u)<0\right\} .
$$


From (3.4) it follows that the set $G(u) \neq \emptyset$ for every $u \in K$. Because the function $A(\cdot, \cdot)+F(\cdot, \cdot)+$ $J^{\circ}(\cdot ; \cdot)$ is convex in the seconde variable, we get that $G(u)$ is a convex set. Now, we prove that for every $v \in K$, the set $G^{-1}(v)=\{u \in K: v \in G(u)\}$ is weakly open. Indeed, from weakly upper semicontinuity of the function

$$
u \mapsto A(T u, T v-T u)+F(u, v-u)+J^{\circ}(S u ; S v-S u)
$$

it follows that

$$
\left[G^{-1}(v)\right]^{c}=\left\{u \in K: A(T u, T v-T u)+F(u, v-u)+J^{\circ}(S u ; S v-S u) \geq 0\right\}
$$

is weakly closed, therefore $G^{-1}(v)$ is weakly open.

Now we verify iii) from Theorem 2.2 i.e. $\bigcup_{v \in K} G^{-1}(v)=K$. Because for every $v \in K$ we have $G^{-1}(v) \subset K$, it follows that $\bigcup_{v \in K} G^{-1}(v) \subset K$. Conversely, let $u \in K$ be fixed. Since $G(u) \neq \emptyset$ there exists $v_{0} \in K$ such that $v_{0} \in G(u)$. In the next step we verify iv) Theorem 2.2. We assert that $D=\bigcap_{v \in K}\left[G^{-1}(v)\right]^{c}$ is empty or weakly compact. Indeed, if $D \neq \emptyset$, then $D$ is a weakly closed set of $K$ since it is the intersection of weakly closed sets. But $K$ is weakly compact hence we get that $D$ is weakly compact. Taking $O_{v}=G^{-1}(v)$ and $K_{0}=K_{1}=K$ we can apply Theorem 2.2 to conclude that there exists $u_{0} \in K$ such that $u_{0} \in G\left(u_{0}\right)$. This give

$$
0=A\left(T u_{0}, T u_{0}-T u_{0}\right)+F\left(u_{0}, u_{0}-u_{0}\right)+J^{0}\left(S u_{0} ; S u_{0}-S u_{0}\right)<0,
$$

which is a contradiction. Therefore the system (QHS) has a solution.

Remark 3.3 If in Theorem 3.1 the sets $K_{i}, i=\overline{1, n}$ are only convex and closed but not bounded we impose the following coercivity condition.

(CC) there exist $K_{i}^{0} \subset K_{i}$ compact sets and $v_{i}^{0} \in K_{i}^{0}$ such that for all $v=\left(v_{1}, \ldots, v_{n}\right) \in K_{1} \times$ $\ldots \times K_{n} \backslash K_{1}^{0} \times \ldots \times K_{n}^{0}$ we have

$$
A\left(T v, T v^{0}-T v\right)+F\left(v, v^{0}-v\right)+\sum_{i=1}^{n} J_{i}^{0}\left(S v, S_{i} v_{i}^{0}-S_{i} v_{i}\right)<0,
$$

where $v^{0}=\left(v_{1}^{0}, \ldots, v_{n}^{0}\right)$. In this case the problem (QHS) has a solution.

\section{Applications}

In this section we are concerned with two applications. In the first application we study the relation between Nash equlibrum and Nash generalized derivative equilibrum points for a hemivariational inequalities system and in the second application we give an existence result for an abstract class of hemivariational inequalities systems.

Let $X_{1}, \ldots, X_{n}$ be Banach spaces and $K_{i} \subset X_{i}$ and the functions $f_{i}: K_{1} \times \cdots \times K_{i} \times \cdots \times K_{n} \rightarrow \mathbb{R}$ for $i \in\{1, \ldots, n\}$. The following notion was introduced by J. Nash [19], 20]:

Definition 4.1 An element $\left(u_{1}^{0}, \ldots, u_{n}^{0}\right) \in K_{1} \times \cdots \times K_{n}$ is Nash equlibrum point of functions $f_{1}, \ldots, f_{n}$ if for each $i \in\{1, \ldots, n\}$ and $\left(u_{1}, \ldots, u_{n}\right) \in K_{1} \times \cdots K_{n}$ we have

$$
f_{i}\left(u_{1}^{0}, \ldots, u_{i}, \ldots, u_{n}^{0}\right) \geq f_{i}\left(u_{1}^{0}, \ldots, u_{i}^{0}, \ldots, u_{n}^{0}\right) .
$$

Now let $D_{i} \subset X_{i}$ be open sets such that $K_{i} \subset D_{i}$ for all $i \in\{1, \ldots, n\}$. We consider the function $f_{i}: K_{1} \times \cdots \times D_{i} \times \cdots K_{n} \rightarrow \mathbb{R}$ which are continuous and locally Lipschitz in the ith variable. The next notion was introduced recently by A. Kristály [13] and is a little bit different form for functions defined on Riemannian manifolds. 
Definition 4.2 If $\left(u_{1}^{0}, \ldots, u_{n}^{0}\right) \in K_{1} \times \cdots \times K_{n}$ is an element such that

$$
f_{i}^{0}\left(u_{1}^{0}, \ldots, u_{n}^{0} ; u_{i}-u_{i}^{0}\right) \geq 0
$$

for every $i=\{1, \ldots, n\}$ and $\left(u_{1}, \ldots, u_{n}\right) \in K_{1} \times \cdots \times K_{n}$ we say that $\left(u_{1}^{0}, \ldots, u_{n}^{0}\right)$ is a Nash generalized derivative points for the functions $f_{1}, \ldots, f_{n}$.

Remark 4.1 If the functions $f_{i}, i \in\{1, \ldots, n\}$ are differentiable in the ith variable, then the above notion coincides with the Nash stationary point introduced in [9].

Remark 4.2 Is is easy to observe that any Nash equlibrum point is a Nash generalized derivative point.

The following result is an existence result for Nash generalized derivative points and is an infinite-dimensional version of a result from the paper [13. Therefore, if in Theorem 3.1] we choose $F_{i}=0, i \in\{1, \ldots, n\}$ and $J=0$ we obtain the following result.

Theorem 4.1 (i) Let $Y_{1}, Y_{2}, \ldots, Y_{n}$ and $X_{1}, X_{2}, \ldots, X_{n}$, be a reflexive Banach spaces and $T_{i}$ : $X_{i} \rightarrow Y_{i}$ compact, linear operators. We consider the closed, convex, bounded sets $K_{i} \subset X_{i}$ and the functions $A_{i}: Y_{1} \times \cdots \times Y_{n} \rightarrow \mathbb{R}, i=1, \ldots, n$ which are locally Lipschitz in the ith variable and satisfies the condition (A). In these conditions, there exists $\left(u_{1}^{0}, \ldots, u_{i}^{0}, \ldots, u_{n}^{0}\right) \in K_{1} \times \cdots \times K_{i} \times$ $\cdots \times K_{n}$ such that for all $i \in\{1, \ldots, n\}$ and $\left(u_{1}, \ldots, u_{i}, \ldots, u_{n}\right) \in K_{1} \times \cdots \times K_{i} \times \cdots \times K_{n}$ we have

$$
A_{i}^{0}\left(\left(T_{1} u_{1}^{0}, \ldots, T_{i} u_{i}^{0}, \ldots, T_{n} u_{n}^{0}\right) ; T_{i} u_{i}-T_{i} u_{i}^{0}\right) \geq 0,
$$

i.e. $\left(u_{1}^{0}, \ldots, u_{i}^{0}, \ldots, u_{n}^{0}\right)$ is a Nash generalized derivative points for the function $A_{i}, i \in\{1, \ldots, n\}$.

(ii) If the sets $K_{i}, i=\{1, \ldots, n\}$ are only closed and convex we suppose that there exists the bounded, closed sets $K_{i}^{0} \subset K_{i}$ and $v_{i}^{0} \in K_{i}^{0}, i=\{1, \ldots, n\}$ such that for every $\left(u_{1}, \ldots, u_{n}\right) \in$ $K_{1} \times \cdots \times K_{n} \backslash K_{1}^{0} \times \cdots \times K_{n}^{0}$ we have

$$
A\left(T u, T v^{0}-T u\right)<0 .
$$

Then there exist $u^{0}=\left(u_{1}^{0}, \ldots, u_{i}^{0}, \ldots, u_{n}^{0}\right) \in K_{1} \times \cdots \times K_{i} \times \cdots \times K_{n}$ such that for all $i \in\{1, \ldots, n\}$ and $u=\left(u_{1}, \ldots, u_{i}, \ldots, u_{n}\right) \in K_{1} \times \cdots \times K_{i} \times \cdots \times K_{n}$ we have

$$
A_{i}^{0}\left(T u_{0} ; T_{i} u_{i}-T_{i} u_{i}^{0}\right) \geq 0,
$$

i.e. $u^{0}=\left(u_{1}^{0}, \ldots, u_{i}^{0}, \ldots, u_{n}^{0}\right)$ is a Nash generalized derivative points for the functions $A_{i}, i \in$ $\{1, \ldots, n\}$.

In the next step we give an existence result for a general system of hemivariational inequalities. In this case in Theorem 3.1 we choose $Y_{i}=Z_{i}, i \in\{1, \ldots, n\}$ and we suppose that the functions $A_{i}: Y_{1} \times \cdots \times Y_{i} \times \cdots \times Y_{n} \rightarrow \mathbb{R}$ are differentiable in the ith variable for $i \in\{1, \ldots, n\}$. In this case we suppose that the functions $A_{i}^{\prime}: Y_{1} \times \cdots \times Y_{i} \times \cdots \times Y_{n} \times Y_{i} \rightarrow \mathbb{R}$ are continuous for $i \in\{1, \ldots, n\}$. Let also $J: Y_{1} \times \cdots \times Y_{i} \times \cdots \times Y_{n} \rightarrow \mathbb{R}$ a locally Lipschitz regular function.

Under these conditions we have the following result.

Corollary 4.1 Let $J, A_{i}: Y_{1} \times \cdots \times Y_{i} \times \cdots \times Y_{n} \rightarrow \mathbb{R}$ be the function as above and suppose that the condition (TS) holds and let $K_{i} \subset X_{i}, i=\{1, \ldots, n\}$ be bounded, closed and convex sets. Under these conditions there exist an element $u^{0}=\left(u_{1}^{0}, \ldots, u_{n}^{0}\right) \in K_{1} \times \cdots \times K_{n}$ such that for every $u=\left(u_{1}, \ldots, u_{n}\right) \in K_{1} \times \cdots \times K_{n}$ and $i \in\{1, \ldots, n\}$ we have:

$$
A_{i}^{\prime}\left(T u^{0} ; T_{i} u_{i}-T_{i} u_{i}^{0}\right)+J_{i}^{0}\left(T u^{0} ; T_{i} u_{i}-T_{i} u_{i}^{0}\right) \geq 0 .
$$


If in Theorem 3.1 we take $A_{i}=0$ then we obtain the following existence result for a general class of hemivariational inequalities systems.

Corollary 4.2 Let $K_{i} \subset X_{i}$ bounded, closed and convex subsets of the reflexive Banach spaces $X_{i}$ for $i \in\{1, \ldots, n\}$. We suppose that $F_{i}: K_{1} \times \cdots \times K_{n} \rightarrow X_{i}^{\star}$ satisfies the condition $(\mathbf{F})$ and $J: Z_{1} \times \cdots \times Z_{n} \rightarrow \mathbb{R}$ is a regular locally Lipschitz function and the condition (TS) holds. Then there exists $u^{0}=\left(u_{1}^{0}, \ldots, u_{i}^{0}, \ldots, u_{n}^{0}\right) \in K_{1} \times \cdots \times K_{i} \times \cdots K_{n}$ such that for every $u=$ $\left(u_{1}, \ldots, u_{i}, \ldots, u_{n}\right) \in K_{1} \times \cdots \times K_{i} \times \cdots K_{n}$ and $i \in\{1, \ldots, n\}$ we have

$$
\left\langle F_{i}\left(u^{0}\right) ; u_{i}-u_{i}^{0}\right\rangle_{i}+J_{i}^{0}\left(S u^{0} ; S_{i} u_{i}-S_{i} u_{i}^{0}\right) \geq 0 .
$$

The above result generalize the main result from the paper of A. Kristály [10].

Indeed, let $\Omega \subset \mathbb{R}^{N}$ be a bounded, open subset. Let $j: \Omega \times \underbrace{\mathbb{R}^{k} \times \cdots \mathbb{R}^{k}}_{n} \rightarrow \mathbb{R}$ a Carathéodory function such that $j(x, \cdot, \ldots, \cdot)$ is locally Lipschitz for every $x \in \Omega$ and satisfies the following assumptions for all $i \in\{1, \ldots, n\}$ :

$\left(j_{i}\right)$ there exists $h_{1}^{i} \in L^{\frac{p}{p-1}}\left(\Omega, \mathbb{R}_{+}\right)$and $h_{2}^{i} \in L^{\infty}\left(\Omega, \mathbb{R}_{+}\right)$such that

$$
\left|z_{i}\right| \leq h_{1}^{i}(x)+h_{2}^{i}(x)|y|_{\mathbb{R}^{k n}}^{p-1}
$$

for almost $x \in \Omega$ and every $y=\left(y_{1}, \ldots, y_{n}\right) \in \underbrace{\mathbb{R}^{k} \times \cdots \times \mathbb{R}^{k}}_{n}$ and $z_{i} \in \partial_{i} j\left(x, y_{1}, \ldots, y_{n}\right)$.

In this case let $S=\left(S_{1}, \ldots, S_{n}\right): X_{1} \times \cdots \times X_{n} \rightarrow L^{p}\left(\Omega, \mathbb{R}^{k}\right) \times \cdots L^{p}\left(\Omega, \mathbb{R}^{k}\right)$ and $J \circ S:$ $K_{1} \times \cdots \times K_{n} \rightarrow \mathbb{R}$ is defined by

$$
J(S u)=\int_{\Omega} j\left(x, S_{1} u_{1}(x), \ldots S_{n} u_{n}(x)\right) d x .
$$

Using a result from Clarke [5] we have:

$$
J_{i}^{0}\left(S u ; S_{i} v_{i}\right) \leq \int_{\Omega} j_{i}^{0}\left(x, S_{1} u_{1}(x), \ldots S_{n} u_{x} ; S_{i} v_{i}(x)\right) d x,
$$

for every $i \in\{1, \ldots, n\}$ and $v_{i} \in X_{i}$.

Therefore we have the following existence result obtained by Kristály [10].

Corollary 4.3 Let $K_{i} \subset X_{i}$ bounded, closed and convex subsets of the reflexive Banach spaces $X_{i}$ for $i \in\{1, \ldots, n\}$. We suppose that $F_{i}: K_{1} \times \cdots \times K_{n} \rightarrow X_{i}^{\star}$ satisfies the condition $(\mathbf{F})$ and $j: \Omega \times \underbrace{\mathbb{R}^{k} \times \cdots \mathbb{R}^{k}}_{n} \rightarrow \mathbb{R}$ a Carathéodory function such that $j(x, \cdot, \ldots, \cdot)$ is a regular, locally Lipschitz function satisfying condition $\left(j_{i}\right)$ and the condition (TS) holds. Then there exists $u^{0}=$ $\left(u_{1}^{0}, \ldots, u_{i}^{0}, \ldots, u_{n}^{0}\right) \in K_{1} \times \cdots \times K_{i} \times \cdots K_{n}$ such that for every $u=\left(u_{1}, \ldots, u_{i}, \ldots, u_{n}\right) \in K_{1} \times$ $\cdots \times K_{i} \times \cdots K_{n}$ and $i \in\{1, \ldots, n\}$ we have

$$
\left\langle F_{i}\left(u^{0}\right) ; u_{i}-u_{i}^{0}\right\rangle_{i}+\int_{\Omega} j_{i}^{0}\left(x, S_{1} u_{1}^{0}(x), \ldots, S_{n} u_{n}^{0}(x) ; S_{i} u_{i}(x)-S_{i} u_{i}^{0}(x)\right) d x \geq 0 .
$$

Remark 4.3 If $n=1$ we obtain a similar result from the paper of Panagiotopoulos, Fundo and Rădulescu [8].

Remark 4.4 If the Banach spaces $X_{i}, i \in\{1, \ldots, n\}$ are separable and the domain $\Omega \subset \mathbb{R}^{N}$ is unbounded then a similar inequality to (I) was proved in the paper Dályai and Varga [6]. Therefore, we can state a similar result as Corollary 4.3 in the case when $\Omega \subset \mathbb{R}^{N}$ is an unbounded domain.

Acknowledgement: Cs. Varga has been supported by Grant CNCSIS PN II ID PCE $2008 \mathrm{nr}$. 501, ID 2162. Both authors were supported by Slovenian Research Agency grants No. P1-02920101 and J1-2057-0101. 


\section{References}

[1] G. Bonanno, G.M. Bisci, D. O'Regan, Infinitely many weak solutions for a class of quasilinear elliptic systems, Mathematical and Computer Modelling 52 (2010), 152-160.

[2] B.E. Breckner, Cs. Varga, A multiplicity result for gradient-type systems with nondifferentiable term, Acta Math. Hungar. 118 (2008), 85-104.

[3] B.E. Breckner, A. Horváth, Cs. Varga, A multiplicity result for a special class of gradient-type systems with non-differentiable term, Nonlinear Anal. TMA, 70(2009), 606-620.

[4] K.-C. Chang, Methods in Nonlinear Analysis, Springer Verlag, Berlin, 2005.

[5] F. H. Clarke, Optimization and Nonsmooth Analysis, SIAM, Philadelphia 1990.

[6] Z. Dályai, Cs. Varga, An existence result for hemivariational inequalities, Electron. J. Differential Equations 37 (2004), 1-17.

[7] K. Fan, Some properties of convex sets related to fixed point theorem, Math. Ann. 266 (1984), 519-537.

[8] P.D. Panagiotopoulos, M. Fundo, V. Rădulescu, Existence Theorems of Hartman-Stampacchia Type for Hemivariational Inequalities and Applications, J. Global Optim. 15 (1999), 41-54.

[9] G. Kassay, J. Kolumbán, Zs. Páles, On Nash Stationary Points, Publ. Math. Debrecen 54 (1999), 267-279.

[10] A. Kristály, Hemivariational inequalities systems and applications, Mathematica 46 (2004), No.2, 161-168.

[11] A. Kristály, An existence result for gradient-type systems with a nondifferentiable term on unbounded strips, J. Math. Anal. Appl. 229 (2004), 186-204.

[12] A. Kristály, Existence of two non-trivial solutions for a class of quasilinear elliptic variational systems on stripe-like domains, Proc. Edinburgh Math. Soc. 48 (2005), 1-13.

[13] A. Kristály, Location of Nash equilibria: a Riemannian geometrical approach, Proc. Amer. Math. Soc. 138 (2010), 1803-1810.

[14] A. Kristály, Cs. Varga, Variational-Hemivariational Inequalities on Unbounded Domains, Studia Univ. Babeş-Bolyai, Mathematica, LV(2010), Nr. 2, 3-87.

[15] A. Kristály, V. Rădulescu, and Cs. Varga, Variational Principles in Mathematical Physics, Geometry, and Economics: Qualitative Analysis of Nonlinear Equations and Unilateral Problems, Encyklopedia of Mathematics (No. 136), Cambridge University Press, Cambridge, 2010.

[16] H. Lisei, Cs. Varga, Multiple solutions for gradient elliptic systems with nonsmooth boundary conditions, Mediterr. J. Math. 8 (2011), 69-79.

[17] D. Motreanu, V. Rădulescu, Variational and non-variational methods in nonlinear analysis and boundary value problems. Nonconvex Optimization and its Applications. Kluwer Academic Publishers, Dordrecht 2003.

[18] D. Motreanu, P. D. Panagiotopoulos, Minimax Theorems and Qualitative Properties of the Solutions of Hemivariational Inequalities, Kluwer Academic Publishers, Dordrecht 1999.

[19] J. Nash, Equilibrium points in n-person games, Proc. Nat. Acad. Sci. USA, 36(1950), 48-49.

[20] J.F. Nash, Non-cooperative games. Ann. of Math. (2) 54 (1951), 286-295. 
[21] P. D. Panagiotopoulos, M. Fundo, V. Rădulescu, Existence Theorems of Hartman-Stampacchia Type for Hemivariational Inequalities and Applications, Journal of Global Optimization 15(1999), 41-54.

[22] E. Tarafdar, Five equivalent theorems on a convex subset of a topological vector space, Commentationes Math. Univ. Carolinae, Vol. 30 (1989), No.2, 323-326.

[23] E. Tarafdar, A fixed point theorem equivalent to the Fan-Knaster-Kuratwski-Mazurkiewicz theorem, Journal Math. Anal. Appl. 128(1987), 475-497. 